\title{
Gradenigo's syndrome
}

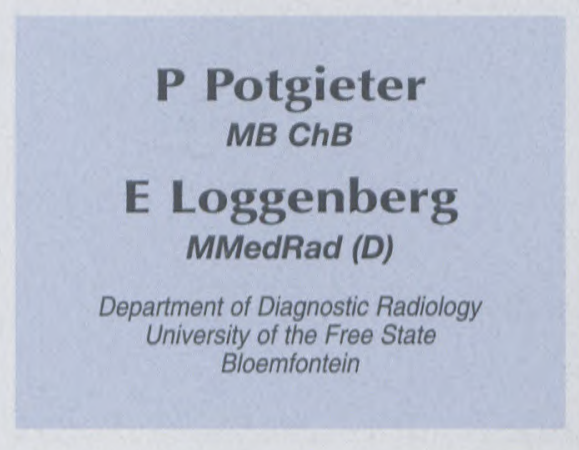

\section{Case report}

A 3-year-old child with a oneweek history of otitis media was treated with intravenous antibiotics in a peripheral hospital. The child then presented with a painful left eye, mild periorbital oedema, diplopia, and otorrhoea from the left ear. On examination the following were found: suppurative otitis media left ear with perforation of the tympanic membrane, musculus rectus lateralis paralysis left eye, facial pain on the left side with N.V1 and N.V2 distribution, and periorbital oedema.

Computed tomography (CT) revealed left mastoid sinus (with air present), and left middle ear and external ear canal opacification. Localised phlebitis was indicated by air in the superior and inferior petrosal venous sinuses, as well as the cavernous sinus on the left side. No bone destruction/osteitis was seen, but there was pneumatisation of petrosal bone (Figs 1 - 3).

Ten-day treatment was given with resolution of the infection and healing of the tympanic membrane. The treatment included the intravenous antibiotics Augmentin (amoxicillin plus clavulanate), metronidazole and chloramphenicol.

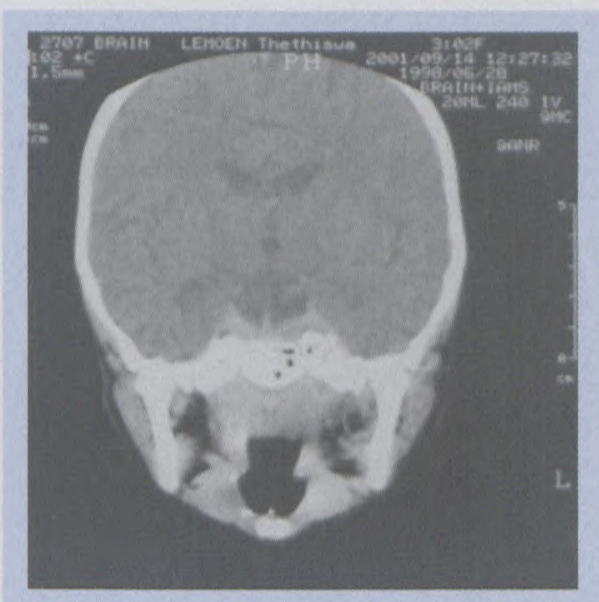

Fig. 1. Coronal of brain with air in petrosal venous sinus.

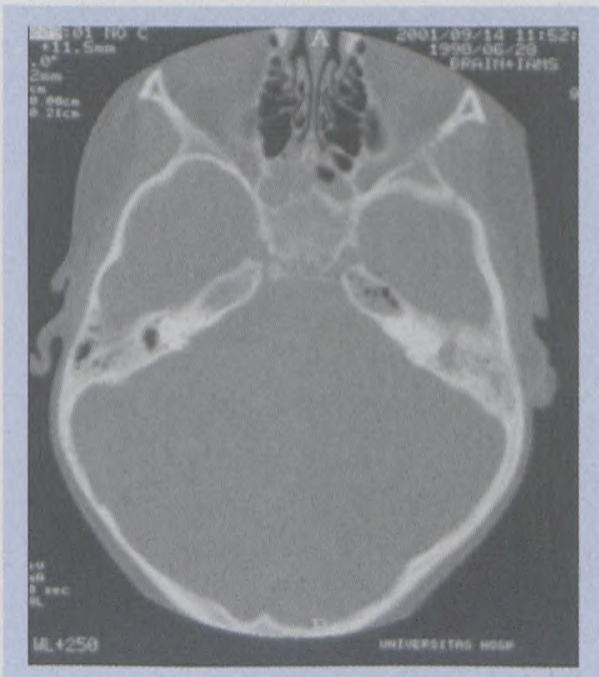

Fig. 2. Axial of brain with air in petrosal venous sinus.

\section{Discussion}

A syndrome characterised by the triad of suppurative otitis media, trigeminal nerve pain in the first divi-

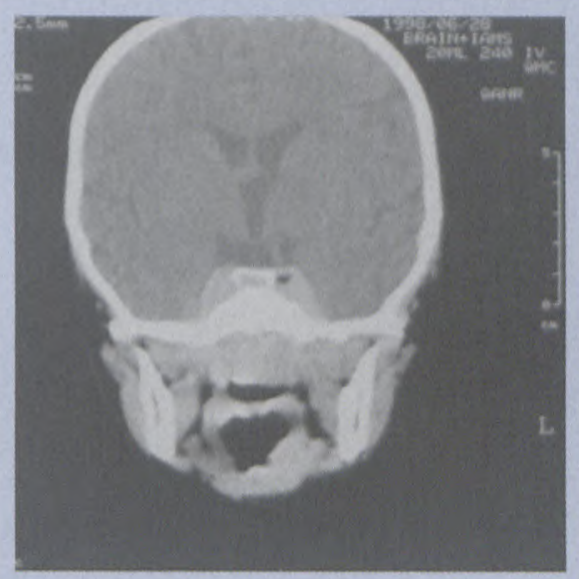

Fig. 3. Coronal of brain with air in cavernous sinus.

sion distribution and abducens nerve (N.V1) palsy was first described in 1907 by Guisseppe Gradenigo.' If not recognised early, the syndrome may have fatal complications. Incidence of the syndrome has declined with the early use of antibiotics, but it is still seen in neglected cases of otitis media. The diagnostic criteria include suppurative otitis media, trigeminal nerve pain (N.V1) and occasionally N.V2 distribution, and abducens nerve palsy presenting as rectus lateralis palsy of the eye.'

Otitis media is a common ear infection. With the early use of antibiotics, complications are relatively mild, with perforation of the tympanic membrane most common. More severe complications may arise if the infection is neglected, or the pathogen is resistant to antibiotics.

The complications include meningitis, intracranial abscess, spread to the skull base with involvement of IX, X, XI cranial nerves (Vernet's syndrome), cavernous sinus involvement, and prevertebral/parapharyngeal abscess. ${ }^{1}$ These complications are presented as: (i) suppurative otitis media with otorrhoea, usually accompanied by mastoiditis; (ii) trigeminal nerve pain due to irritation of the gasserian 


\section{CASE REPORT}

Abducens palsy with petrous apicitis

\section{CT of brain and HRCT of temporal bones}

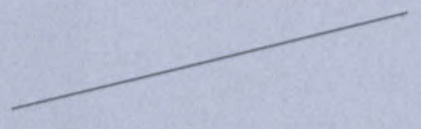

Bone erosion or history of chronic ear disease
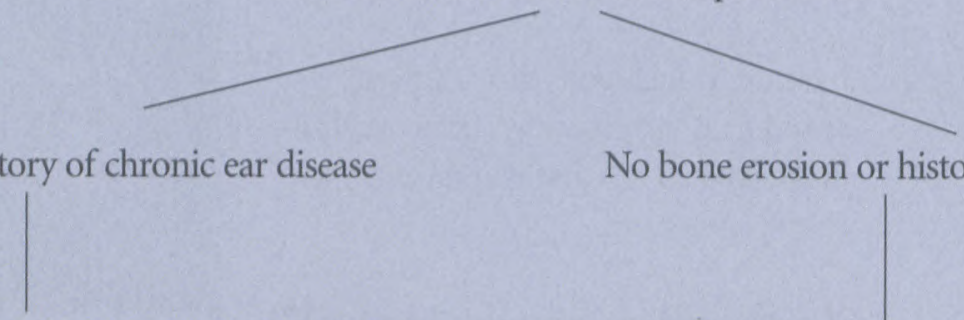

1. Culture directed IV antibiotics

No bone erosion or history of chronic ear disease

2. Mastoidectomy with drainage of petrous apices

1. Culture directed IV antibiotics

2. Myringotomy

Fig. 4. Algorithm of management of patients with nervus abducens palsy.

ganglion situated in Meckel's cave; (iii) abducens nerve palsy presents as $\mathrm{m}$. rectus lateralis weakness/palsy of the eye due to compression of the nerve in Dorello's canal, a dural sheath at the petrosphenoidal ligament; (iv) periorbital oedema may also be a presenting symptom; and $(v)$ retrobulbar pain.

The infection may spread via pneumatised air cell tracts, through vascular channels, or direct extension through facial planes. The infection may spread to the petrous apex of the temporal bone, giving rise to apical petrositis in patients with suppurative otitis media.

Petrositis may be acute or chronic, depending on the clinical course and severity of the disease. Fig. 4 represents a general regimen for approaching a patient with N.V1 palsy. ${ }^{2}$ Pneumatisation plays an important role in the spread of the disease throughout the mastoid and petrous bone, but infection and inflammation may also spread to areas of non-pneumatised petrous bone by way of direct extension or venous channels.

In this case study, involvement of the cavernous sinus added a twist as to how the infection could spread and the complications that could follow if the patient was not examined and treated properly.

\section{References}

1. Motamed M, Kalan A. Gradenigo's syndrome Postgrad Med J 2000: 76: 559-560.

2. Minotti AM, Kountakes SE. Management of abducens palsy in patients with petrositis. Ann Otol Rhinol Laryngol Suppl 1999; 108: 897-902. 